Letter to the Editors-in-Chief

\title{
Peripheral circulating cells with paroxysmal nocturnal haemoglobinuria phenotype after a first episode of cerebral sinus vein thrombosis: Results from a multicentre cross-sectional study
}

A R T I C L E I N F O

\section{Keywords:}

Cerebral sinus venous thrombosis

PNH

Risk factor

Screening

Venous thrombosis

Paroxysmal nocturnal haemoglobinuria (PNH) is a rare, potentially fatal disorder of haematopoietic stem cells caused by mutations in an Xlinked gene called phosphatidylinositol glycan class A, characterised by intravascular haemolysis, bone marrow failure and thrombotic events. The disease can occur at any age, although preferentially it affects young adults; its estimated prevalence is about $1 / 500,000$ [1].

Clinical symptoms are variable and can include haemolytic anaemia, moderate to severe impairment of haematopoiesis and, in approximately $40 \%$ of patients, thrombosis of the vessels of the abdomen, brain and skin [2]. Rare, atypical site thrombosis of the splanchnic veins or cerebral sinuses are recognized as suggestive of the presence of a PNH clone [3]. To date, however, the prevalence of PNH clones in unselected patients with cerebral venous thrombosis is not yet known. The primary objective of this study was to determine the prevalence of PNH clones in patients with a recent history of cerebral sinus venous thrombosis (CSVT) and up to twenty-four months follow-up. In this multicentre cross-sectional study, patients with an objective diagnosis of CSVT of new onset or occurring in the previous three years were investigated for the presence of PNH clones. Known or clinically suspected PNH and active treatments able to interfere with the laboratory assessment of a PNH clone (e.g., recent chemotherapy, treatment with hypomethylating drugs or immunosuppressive therapy) were exclusion criteria, patients with a family history of PNH were also excluded, whereas patients treated with erythroid and/or granulocyte growth factors were eligible for this study. CSVT diagnosis was objectively obtained by one or more of the following imaging tests: computed tomography, magnetic resonance imaging or cerebral angiography. After written informed consent was obtained, the following data for each enrolled patient were collected from patient charts: age; gender; race; personal or family history of venous thromboembolism (VTE); the presence of inherited [antithrombin, protein C or protein S deficiency; Factor V Leiden gene mutations; Factor II (G20210A) gene mutations; increased coagulation Factor VIII levels; increased homocysteine levels] or acquired thrombophilia states (i.e., lupus anticoagulant, anticardiolipin antibodies, beta2-glycoprotein 1 antibodies); known recurrence of CSVT; history of Philadelphia-negative myeloproliferative neoplasm; detection of JAK2 (V617F) mutation (even without overt myeloproliferative neoplasm); other known risk factors for CSVT such as cancer, pregnancy, childbirth, use of oral contraceptives, parameningeal infections; other known haematological disorders; systemic lupus erythematosus (SLE); Behçet's disease, nephrotic syndrome and previous lumbar punctures for diagnostic or therapeutic aims. Patients were followed up to two years after diagnosis. Each abnormal test result was confirmed in a second assay. Testing for PNH clones was undertaken at each enrolling center and then centrally confirmed at the laboratory of the Haematology Unit of the University Hospital "Vittorio Emanuele", Catania.

The presence of a PNH clone was assessed by multi-parameter flow cytometric analysis [4].

The test was performed on neutrophil granulocytes, monocytes and erythrocytes, using peripheral blood samples collected in EDTA Vacutainer ${ }^{\circledR}$ tubes. All samples were processed within $24 \mathrm{~h}$ of their arrival at the central laboratory (from 24 to $48 \mathrm{~h}$ after blood sampling). For the evaluation on neutrophil granulocytes and monocytes, a single five-colour reagent combination was adopted as follows: FLAER, CD24PE, CD45-ECD, CD33 PE-cy5 and CD14 PE-cy7. A multi-parameter gating strategy was used by combining CD45 and CD33 expression (non-GPI-linked molecules) to identify the granulocyte and monocyte populations, and on these was valuated the expression of two different GPI-linked molecules (FLAER/CD24 on granulocytes and FLAER/CD14 on monocytes).

The study was conducted in accordance with the Declaration of Helsinki, and Ethics Committee approval was obtained at each enrolling centre. A sample of convenience of at least 70 patients was deemed sufficient to detect at least one positive sample if the true prevalence of PNH clones in asymptomatic subjects was $0.7 \%$ in the regions participating to the study [5]). Descriptive statistics were adopted for the purpose of the current study. Data were collected by each enrolling physician in a dedicated database hosted at the University Hospital Vittorio Emanuele, Catania. Seventy-seven subjects were enrolled (40 females, 37 males, mean age, 41 years, range 19-87) from the following four Regional Centres for Thrombosis and Haemostasis: "P.Giaccone", Palermo; "Vittorio Emanuele", Catania; "Pugliese Ciaccio", Catanzaro and "Morelli", Reggio Calabria (Fig. 1).The study started in January 2016 and closed in January 2019, enrolment lasted 12 months. Anticoagulant treatment for CSVT (Table 1) was administered for a mean of 


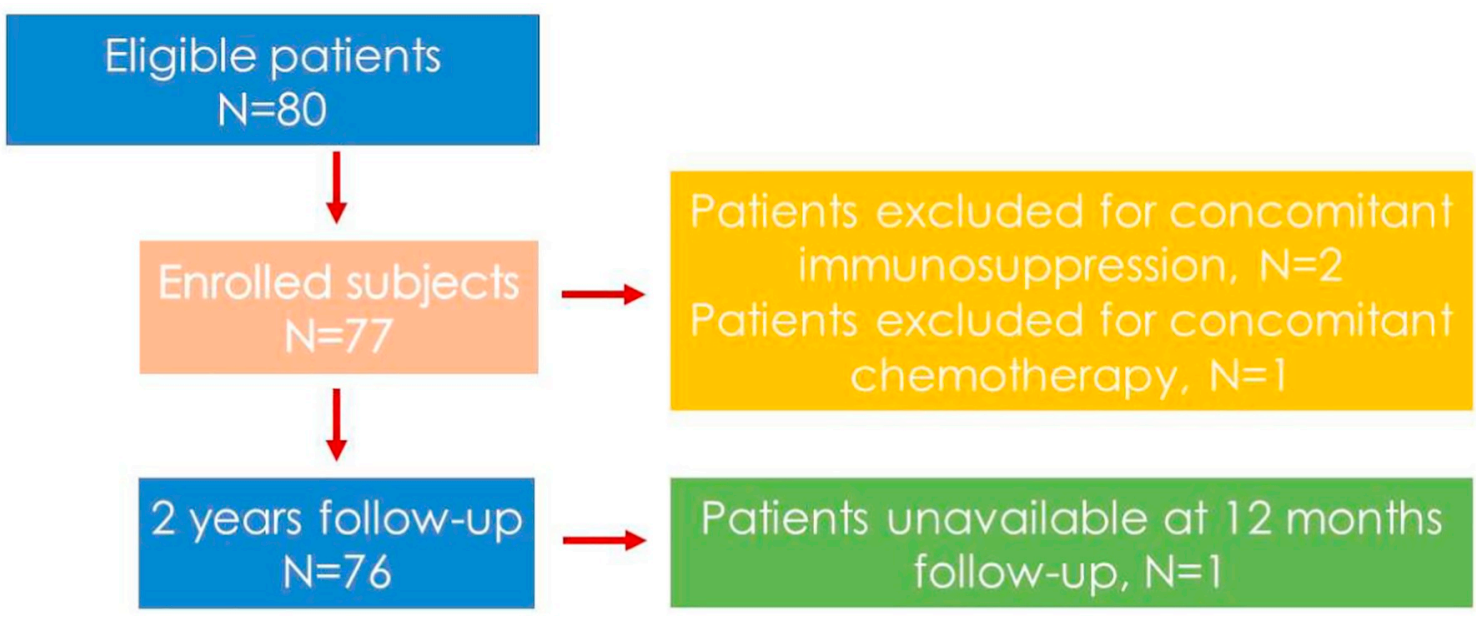

Fig. 1. Study flow chart.

Table 1

Demographic characteristics, risk factors and anticoagulant treatment of the enrolled patients.

\begin{tabular}{ll}
\hline Variable & \\
\hline Number & 77 \\
Age, mean (range) & 41 years (19-87) \\
Males, mean (range) & $37(19-74)$ \\
Females, mean (range) & $40(24-87)$ \\
BMI, mean (range) & $23(18-27)$ \\
Anticoagulant treatment duration, months; mean (range) & $6(4-13)$ \\
Non-HDL-c levels; mean (range) & $135(110-160)$ \\
Glucose levels; mean (range) & $92(80-105)$ \\
Alcohol intake; number (\%) & $18(23.4)$ \\
Smoking habit; number (\%) & $32(41.5)$ \\
Hypertension; number (\%) & $28(36.4)$ \\
Chronic kidney disease; number (\%) & $6(7.8)$ \\
Concomitant medications; number (\%) & $30(38.9)$ \\
Anticoagulant treatment with warfarin; number (\%) & $48(62.3)$ \\
Low molecular weight heparin; number (\%) & $29(37.6)$ \\
Patients with factor v Leiden gene mutation at heterozygous & $5(6.5)$ \\
$\quad$ state; number (\%) & \\
Patients with FII (g20210a) gene mutation at heterozygous & $6(7.8)$ \\
$\quad$ state; number (\%) & \\
Patients with jak2 (v6167f) gene mutation; number (\%) & $2(2.5)$ \\
Patients with protein s deficiency; number (\%) & $1(1.2)$ \\
Patients with lupus anticoagulant and anti-phospholipid & $5(6.4)$ \\
$\quad$ antibodies; number (\%) & $38(49.3)$ \\
Patients without risk factor for venous thromboembolism; & \\
$\quad$ number \%) & $2(2.5)$ \\
Patients with PNH clones; number (\%) & \\
\hline
\end{tabular}

BMI = body mass index; non-HDL-C = non-HDL cholesterol (mg/dL); glucose levels refer to fasting glucose $(\mathrm{mg} / \mathrm{dL})$.

six months (range: 4-13 months). Adverse events related to anticoagulant therapy were not reported. CSVT was associated with factor V Leiden gene mutation in the heterozygous state in five $(6.5 \%)$ patients, FII (G20210A) gene mutation in the heterozygous state in six $(7.8 \%)$ patients, JAK2 (V6167F) mutation in two (2.5\%) patients, protein $S$ deficiency in one $(1.2 \%)$ case, lupus anticoagulant and anti-cardiolipin antibodies in five (6.5\%) cases, combined inherited and acquired risk factors were detected in three $(3.8 \%)$ cases (heterozygous FII gene mutation and recent surgery in 2 cases, FV gene mutation and oral contraceptives in 1 case), recent surgery in $10.2 \%$, oral contraceptives in $6.4 \%$ and pregnancy in $3.8 \%$ of cases. Among screened patients, 38 $(49.3 \%)$ did not have any of the aforementioned reported risk factors for VTE. The incidence of PNH clones observed in our cohort was $2.5 \%$. PNH clones were detected in two cases with no other concomitant risk factors. In one patient, a small one (size $0.08 \%$ ) was not further confirmed at centralised determination; in another case, a clearly demonstrable clone (size $1.5 \%$ ) was confirmed on two consecutive independent determinations. The patient with a confirmed PNH clone (a 27-year-old Caucasian female) had a positive familial history of VTE and an otherwise unremarkable personal thrombophilia screening, in absence of any symptom or other laboratory signs of PNH. Two venous thrombosis recurrences (deep vein thrombosis of the legs) occurred at the two-years follow-up, both cases without any evidence of PNH clones. Thrombosis is the main cause of death in patients with PNH [6] and can involve both arterial and venous sites, although the latter are involved in $80-85 \%$ of cases. Even patients who do not develop haemolysis or have only smaller PNH clones have been reported to be at risk of thrombosis [7]. Several mechanisms have been advocated to explain this risk: the release of micro-particles by complement-mediated platelet activation; chronic hypo fibrinolysis due to an altered plasminogen activation; release of free haemoglobin by chronic haemolysis [8]. Thrombosis-related genes, involved in the control of vascular wall integrity, blood flow and thrombosis prevention have been recently explored by whole exome sequencing to better understand the pathogenesis of thrombosis in PNH. Study results support the role of several genes in the pathogenesis of thrombosis, however specific mechanisms still need to be clarified [9].

Available data on CSVT in PNH are lacking and mainly limited to case reports or series [10], showing a high prevalence of CSVT in young subjects with concomitant risk factors and an overall good prognosis when the recognition and treatment of $\mathrm{PNH}$ is prompt. The objective of the current study was to evaluate the prevalence of PNH clones in patients with a clinical history of CSVT. Among 77 patients screened, we did not find the presence of a significant PNH clone; however, we found two small PNH-positive clones, for an incidence of $2.5 \%$. We did not perform an analysis of thrombosis-related genes but this would have been useful particularly in the subject with a PNH clone and a positive family history of VTE.

The absence of a control group is the main limitation of our study since little information exists on the prevalence of small PNH clones in healthy patients, however we are confident in our findings for several reasons: reported frequency of PNH cells in normal subjects is extremely low and the prevalence of clones higher than $0.01 \%$, although unknown, could be expected to be extremely rare; furthermore we have here ruled out transient clones by repeated testing. It is still a matter of debate whether the detection of a small PNH clone can be considered as risk factor for thrombosis; however, small PNH clones have been reported as transient in the general population. The clinical implication of stable small PNH clones deserves further investigations, this is especially important to clarify whether or not the detection of PNH clones, in the absence of other signs and symptoms, suggests PNH and specific treatments beyond anticoagulation. 


\section{Authors' contributions}

MN wrote the paper. GG conceived the study. GS, RCS, SR and LP enrolled patients; GR and AT performed laboratory tests; DN and VC analysed the data and performed the statistical analysis. SS critically revised the manuscript.

\section{Declaration of competing interest}

None of the authors have relevant conflicts of interest to declare.

\section{Acknowledgements}

Authors are grateful to Elsevier language editing service for English language revision.

\section{Appendix A. Supplementary data}

Supplementary data to this article can be found online at https:// doi.org/10.1016/j.thromres.2019.11.025.

\section{References}

[1] P. Hillmen, S.M. Lewis, M. Bessler, L. Luzzatto, J.V. Dacie, Natural history of paroxysmal nocturnal hemoglobinuria, N. Engl. J. Med. 333 (19) (1995) 1253-1258.

[2] A. Hill, R. Kelly, P. Hillmen, Thrombosis in paroxysmal nocturnal hemoglobinuria, Blood. Blood. 121 (25) (2013) 4985-4996.

[3] U.K. Misra, J. Kalita, V. Bansal, P.P. Nair, Paroxysmal nocturnal haemoglobinuria presenting as cerebral venous sinus thrombosis, Transfus. Med. 18 (2008) 308-311.

[4] D.R. Sutherland, M. Keeney, A. Illingworth, Practical guidelines for the high-sensitivity detection and monitoring of paroxysmal Nocturnal Hemoglobinuria Clones by Flow Cytometry, Cytometry B Clin Cytom. 82 (2012) 195-208.

[5] Anita Hill, Philip J. Platts, Alex Smith, Stephen J. Richards, Matthew J. Cullen, et al., The incidence and prevalence of paroxysmal nocturnal hemoglobinuria (PNH) and survival of patients in Yorkshire, Blood 108 (2006) 985.
[6] A. Malato, G. Saccullo, L. Lo Coco, S. Mancuso, M. Santoro, S. Martino, et al., Thrombotic complications in paroxysmal nocturnal haemoglobinuria: a literature review, Blood Transfus. 10 (2012) 428-435.

[7] P. Hillmen, P. Muus, U. Duhrsen, A.M. Risitano, J. Schubert, et al., Effect of the complement inhibitor eculizumab on thromboembolism in patients with paroxysmal nocturnal hemoglobinuria, Blood. 110 (2007) 4123-4128.

[8] W. Ageno, F. Dentali, V. De Stefano, S. Barco, T. Lerede, M. Bazzan, et al., Clonal populations of hematopoietic cells with paroxysmal nocturnal hemoglobinuria phenotype in patients with splanchnic vein thrombosis, Thromb. Res. 133 (2014) 1052-1055.

[9] L. Li, H. Wang, H. Liu, Z. Liu, L. Li, K. Ding, et al., Gene mutations associated with thrombosis detected by whole-exome sequencing in paroxysmal nocturnal hemoglobinuria, Int J Lab Hematol. 41 (3) (2019 Jun) 424-432.

[10] E. Meppiel, I. Crassard, R. Peffault de Latour, S. de Guibert, L. Terriou, H. Chabriat, et al., Cerebral venous thrombosis in paroxysmal nocturnal haemoglobinuria: a series of 15 cases and review of the literature, Medicine (Baltimore). 94 (1) (2015 Jan) e362.

Mariasanta Napolitano ${ }^{\mathrm{a}, *}$, Rita Carlotta Santoro ${ }^{\mathrm{b}}$, Daniela Nicolosi ${ }^{\mathrm{c}}$, Valeria Calafiore ${ }^{\mathrm{c}}$, Anna Triolo ${ }^{\mathrm{d}}$, Simona Raso ${ }^{\mathrm{e}}$, Laura Parrinello ${ }^{\mathrm{d}}$, Giuseppina Rizzo $^{\mathrm{d}}$, Gianluca Sottilotta ${ }^{\mathrm{f}}$, Sergio Siragusa ${ }^{\mathrm{a}}$, Gaetano Giuffrida ${ }^{\mathrm{c}}$

${ }^{\text {a }}$ University of Palermo, PROMISE Department, Haematology Unit and Reference Regional Centre for Thrombosis and Haemostasis, Italy

${ }^{\mathrm{b}}$ Haemophilia, Haemostasis and Thrombosis Centre, AO Pugliese Ciaccio, Catanzaro, Italy

${ }^{\mathrm{c}}$ Division of Haematology, A.O.U. Policlinico Vittorio Emanuele, Catania,

${ }^{\mathrm{d}}$ Cytometryc Lab, Division of Haematology, A.O.U. Policlinico Vittorio Emanuele, Catania, Italy

${ }^{\mathrm{e}}$ Division of Haematology, Department of Surgical, Oncological and Stomatological Disciplines (Di.Chir.On.S.), AOUP Paolo Giaccone, Palermo, Italy

${ }^{\mathrm{f}}$ Haemophilia Centre, Haemostasis and Thrombosis Hospital Morelli, Reggio Calabria, Italy

E-mail address: mariasanta.napolitano@unipa.it (M. Napolitano).

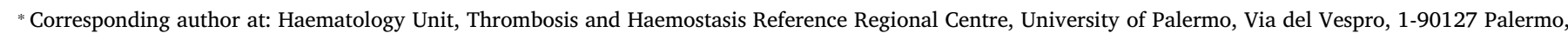
Italy. 